\title{
Results of a survey in Andalusian Endoscopy Units
}

\author{
J. M. Herrerías Gutiérrez, F. Argüelles Arias and Andalusian Team of Chiefs of Gastroenterology Units \\ and Departments ${ }^{1}$
}

Gastroenterology Department. University Hospital "Virgen Macarena”. Seville, Spain

\begin{abstract}
Introduction: in Spain, there is no indication of the resources that an endoscopy unit should have. As a consequence only the experience of professionals who have been working for years in this field can be used as a guide. However, there are some recent surveys on sedation in Spain that indicate how sedation is use in other units.

Objectives: the objectives of the present study were: a) to find out the human and material resources available in endoscopy units in Andalusia; b) to analyze the number of endoscopies performed in our country; and c) to identify factors that can be improved.

Material and methods: in order to make an assessment of the conditions and resources of said units in Andalusia, a survey was designed for the staff of gasteroenterology department. Hospitals involved are those specified in this paper. The survey, which included 90 questions, covers all aspects concerning each unit. There are questions on the real conditions of the unit, and on what would be considered as ideal or at least as minimal requirement for each hospital or Health Area.
\end{abstract}

Conclusions: even though some shortcomings were found in some hospitals, a high percentage of them have acceptable equipment. However, due to fast advances in the field of endoscopy, an

'Juan Esteban Carretero, Hospital Torrecárdenas (Almería); Leopoldo Martín Herrera, Hospital Puerta del Mar (Cádiz); José Manuel Montero Domínguez, Hospital Puerto Real (Cádiz); Juan de Dios Vega, Hospital Reina Sofía (Córdoba); Javier Salmerón Escobar, Hospital Clin. Un. San Cecilio (Granada); Rafael Martín-Vivaldi Martínez, Hospital Virgen de las Nieves (Granada); Agustín Domínguez Macías, Hospital Juan Ramón Jiménez (Huelva); Benito Sánchez Alcalá, Complejo Hospitalario de Jaén (Jaén) Eduardo Franquelo Villalonga, Hospital Clínico Virgen de la Victoria (Málaga); Jorge Maldonado Eloy-García, Hospital Regional Carlos Haya (Málaga); Andrés Manuel Sánchez Cantos, Hospital Costa del Sol (Marbella, Málaga); Manuel Romero Gómez y Manuel Castro Fernández, Hospital Nuestra Señora de Valme (Sevilla); José Luis Márquez Galán, Hospital Universitario Virgen del Rocío (Sevilla); Juan Manuel Herrerías Gutiérrez, Hospital Universitario Virgen Macarena (Sevilla).

Recibido: 11-04-06.

Aceptado: 25-09-06.

Correspondencia: J. M. Herrerías Gutiérrez. Departamento de Aparato Digestivo. Hospital Universitario Virgen Macarena. Sevilla. Tel.: 955008 801. Fax: 955008 805. e-mail: jmhg@us.es improvement in the conditions should be provided, especially in aspects concerning anesthesia.

Key words: Endoscopy. Sedation. Endoscopy Unit. Anesthesia. Accesory material in Endoscopy Units. Survey in Endoscopy Units.

Herrerías Gutiérrez JM, Argüelles Arias F and Andalusian Team of Chiefs of Gastroenterology Units and Departments. Results of a survey in Andalusian Endoscopy Units. Título. Rev Esp Enferm Dig 2006; 98: 929-948.

\section{INTRODUCTION}

Over the last few years, all medical specialties have experienced an extraordinary development. The specialty of Gastroenterology, and specifically its subclass of Endoscopy, has been most affected by new advances. Today, endoscopy is one of the complementary examinations most frequently requested by doctors. This practice cannot be conceived without its therapeutic side, increasingly richer and more varied every day. Its use is increasing because of extended indications, including colorectal cancer screening, and the growing frequency of new therapeutic techniques like ERCP and echoendoscopy.

For these reasons it seems indispensable that endoscopy units be able to face the increasing demand in a fast, safe, efficient way be set up. Since advances develop so quickly, it is necessary to have the foundations to carry out this technique. That is, endoscopy units with more complex endoscopic material, more numerous, more specialized personnel, and a wider coverage.

Several recommendations can be found in the specialized literature concerning sedation requirements in en- 
doscopy units $(1,2)$, as well as some reviews gaining a deeper insight in this topic (3) -a useful guide to find out resources needed by each endoscopy unit in every hospital. In some cases, only the experience of professionals can be used as a working guide satisfactorily. Yet there are some recent surveys in Spain concerning sedation in endoscopy that may shed light on how sedation is being used in other units $(4,5)$.

\section{OBJECTIVES}

The primary aims of this study were:

1. To identify personnel working in contact with endoscopy units in Andalusia.

2. To recognized materials and rooms used in these units.

3. To identify number of endoscopies and the way these studies are performed: sedation, drugs used, time spent in each examination.

As secondary objectives we wished:

1. To find out what units consider necessary for their activity.

2. To know which aspects of the units can be improved.

\section{MATERIAL AND METHODS}

In order to assess the condition of these units in Andalusia, a survey was designed at a meeting held in Antequera, in May 2005, with the participation of most department heads from Andalusian Hospitals. Such survey should be answer by all gastroenterology departments in order to assess their resources and to identify aspects in need of improvement.

Participating hospitals included were: Hospital of Torrecárdenas (Almería), Hospital Virgen de Valme (Seville), Hospital Virgen del Rocío (Seville), Hospital Juan Ramón Jiménez (Huelva), Hospital Reina Sofía (Córdoba), Hospital Costa del Sol (Marbella), Hospital Puerta del Mar (Cádiz), Hospital San Cecilio (Granada), Hospital Virgen de las Nieves (Granada), Hospital Carlos Haya (Málaga), Hospital Virgen de la Victoria (Málaga), Hospital of Jaén, Hospital of Puerto Real, Hospital Virgen Macarena (Seville).

The survey consisted of 90 questions trying to cover all aspects in an endoscopy unit. The first questions involved the actual state of the unit, and the remaining concerned the indispensable resources and ideal situations for every hospital or health area. The aim of the survey was to find out whether Andalusian units have all necessary resources to cover their demand.

The survey was developed by the group during the meeting, and was distributed via email. The deadline for unit replies was one month, and answers included belong to those who answered within such period. Only one hospital failed to respond (93.3\% of Andalusian Team of Chiefs of Gastroenterology Units and Departments was included).

\section{Statistical study}

This was a prospective study based on a survey including 90 questions, where a descriptive statistical study is only used for the analysis of several quantitative variables.

The items included in this survey were divided into several groups and are summarized in table I.

\section{Table I}

Section 1: general questions

1. How many users does your endoscopy unit cover? Fewer than 100,000 people; Between 100,000 and 250,000 people; Between 250,000 and 400,000 people; More than 400,000 people

2. How many people should your unit cover? Fewer than 100,000 people; Between 100,000 and 250,000 people; Between 250,000 and 400,000 people; More than 400,000 people

3. Is it an independent unit or annexed to some department? Independent; Annexed

4. What is ideal -an independent or an annexed unit? Independent; Annexed

Section 2: unit staff

5. How many doctors, both endoscopists and non-endoscopists, work in your unit?

Fewer than 5 doctors; Between 5 and 10 doctors; Between 10 and 15 doctors; More than 15 doctors

6. What is the ideal number of doctors, endoscopists and non-endoscopists, for your unit?

Fewer than 5 doctors; Between 5 and 10 doctors; Between 10 and 15 doctors; More than 15 doctors

7. How many doctors work full-time in your unit? None: < 33\%; 33-66\%; > 66\%

8. How many doctors do you think that should work full-time in your unit? None; <33\%; 33-66\%; > 66\%

9. How many nurses are there in your endoscopy unit? Fewer than 2 nurses; Between 2 and 4 nurses; Between 4 and 6 nurses; More than 6 nurses

10. What do you think the ideal number of nurses in an endoscopy unit would be?

Fewer than 2 nurses; Between 2 and 4 nurses; Between 4 and 6 nurses; More than 6 nurses

11. How many healthcare assistants are there in your endoscopy unit? Fewer than 2 healthcare assistants; Between 2 and 4 healthcare assistants; Between 4 and 6 healthcare assistants; More than 6 healthcare assistants

12. What do you think the ideal number of health care assistants in an endoscopy unit would be?

Fewer than 2 healthcare assistants; Between 2 and 4 healthcare assistants; Between 4 and 6 healthcare assistants; More than 6 healthcare assistants

Section 3: unit materials

13. How many operating rooms are there in you endoscopy unit? One; Two; Three; Four or more

14. What is the ideal number of operating rooms for your endoscopy unit? One; Two; Three; Four or more

15. How many endoscopes are there in your endoscopy unit? Fewer than 5 endoscopes; Between 5 and 10 endoscopes; Between 10 and 15 endoscopes; More than 15 endoscopes

16. What is the ideal number of endoscopes for your endoscopy unit? Fewer than 5 endoscopes; Between 5 and 10 endoscopes; Between 10 and 15 endoscopes; More than 15 endoscopes

(see following page) 


\section{Table I (Cont.)}

17. How many gastroscopes are there in your endoscopy unit? Fewer than 2 gastroscopes; Between 2 and 4 gastroscopes; Between 4 and 6 gastroscopes; More than 6 gastroscopes

18. What is the ideal number of gastroscopes for your endoscopy unit? Fewer than 2 gastroscopes; Between 2 and 4 gastroscopes; Between 4 and 6 gastroscopes; More than 6 gastroscopes

19. What is the number of colonoscopes in you endoscopy unit? Fewer than 2 colonoscopes; Between 2 and 4 colonoscopes; Between 4 and 6 colonoscopes; More than 6 colonoscopes

20. What is the ideal number of colonoscopes for your endoscopy unit? Fewer than 2 colonoscopes; Between 2 and 4 colonoscopes; Between 4 and 6 colonoscopes; More than 6 colonoscopes

21. How many duodenoscopes are there in your endoscopy unit? One; Two; Three; Four or more

22. What is the ideal number of duodenoscopes for your endoscopy unit? One; Two; Three; Four or more

23. How many enteroscopes are there in your endoscopy unit? None; One; Two; Three or more

24. What is the ideal number of enteroscopes for your endoscopy unit? None; One; Two; Three or more

25. How many echoendoscopes are there in your endoscopy unit? None; One; Two; Three or more

26. What is the ideal number of echoendoscopes for your endoscopy unit? None; One; Two; Three or more

27. How many upper endoscopies are carried out in your endoscopy unit on a daily basis?

Fewer than 10 upper endoscopies; Between 10 and 20 upper endoscopies; Between 20 and 30 upper endoscopies; More than 30 upper endoscopies

28. What is the ideal number of daily upper endoscopies for your endoscopy unit?

Fewer than 10 upper endoscopies; Between 10 and 20 upper endoscopies; Between 20 and 30 upper endoscopies; More than 30 upper endoscopies

29. How many colonoscopies are performed daily in your endoscopy unit? Fewer than 10 colonoscopies; Between 10 and 20 colonoscopies; Between 20 and 30 colonoscopies; More than 30 colonoscopies

30. How many colonoscopies should be ideally performed daily in your endoscopy unit?

Fewer than 10 colonoscopies; Between 10 and 20 colonoscopies; Between 20 and 30 colonoscopies; More than 30 colonoscopies

31. How many enteroscopies are performed weekly in your endoscopy unit? None; One; Two; Three or more

32. How many enteroscopies should be ideally performed weekly in your endoscopy unit?

None; One; Two; Three or more

33. How many ERCPs are performed weekly in your endoscopy unit? None; One; Two; Three or more

34. What is the number of ERCPs that should be ideally performed every week in your endoscopy unit? None; One; Two; Three or more

35. How many echoendoscopies are performed in your endoscopy unit every on a weekly basis? None; One; Two; Three or more

36. How many echoendoscopies should be ideally performed in your endoscopy unit every week? None; One; Two; Three or more

37. How many polypectomies are performed in your endoscopy unit every week?

None; One; Two; Three or more

38. How many polypectomies should be ideally performed in your endoscopy unit every week?

None; One; Two; Three or more

39. How many endoscopic treatments (sclerotherapy or ligation) of varicose veins are performed in your endoscopy unit every week?

None; One; Two; Three or more
40. How many endoscopic treatments (sclerotherapy or ligation) should be ideally performed in your endoscopy unit every week?

None; One; Two; Three or more

41. How many hemorrhoidal treatments are performed in your endoscopy unit every week?

None; One; Two; Three or more

42. How many hemorrhoidal treatments should be ideally performed in your endoscopy unit every week?

None; One; Two; Three or more

43. How many treatments with argon beam are performed in your endoscopy unit every week?

None; One; Two; Three or more

44. How many treatments with argon beam should be ideally performed in your endoscopy unit every week? None; One; Two; Three or more

45. How many palliative endoscopic treatments are performed in your endoscopy unit every week? None; One; Two; Three or more

46. How many palliative endoscopic treatments should be ideally performed in your endoscopy unit every week?

None; One; Two; Three or more

47. What is the estimated time between upper endoscopies in your endoscopy unit?

Less than 15 minutes; 15-25 minutes; 25-35 minutes; More than 35 minutes

48. What is the ideal estimated time between upper endoscopies for you endoscopy unit?

Less than 15 minutes; 15-25 minutes; 25-35 minutes; More than 35 minutes

49. What is the estimated time between colonoscopies in your endoscopy unit?

Less than 25 minutes; 25-40 minutes; 40-55 minutes; More than 55 minutes

50. What is the ideal estimated time between colonoscopies for your endoscopy unit?

Less than 25 minutes; 25-40 minutes; 40-55 minutes.More than 55 minutes

51. What is the estimated time for a non-basic examination (enteroscopy; echoendoscopy, ERCP, endoscopic therapy) in your endoscopy unit? Less than 40 minutes; 40-70 minutes; 70-100 minutes; More than 100 minutes

52. What is the ideal estimated time for a non-basic examination (enteroscopy, echoendoscopy, ERCP, endoscopic therapy) for your endoscopy unit?

Less than 40 minutes; 40-70 minutes; 70-100 minutes; More than 100 minutes

Section 4: sedation and anesthesia

53. How many upper digestive endoscopies are performed in your unit using sedation?

None; <33\%; 33-66\%; > 66\%

54. How many upper digestive endoscopies should be ideally performed in your unit using sedation?

None; $<33 \%$; $33-66 \%$; $>66 \%$

55. How many colonoscopies are performed in your unit using sedation? None; $<33 \%$; 33-66\%; $>66 \%$

56. How many colonoscopies should be ideally performed in your unit using sedation?

None; $<33 \%$; 33-66\%; > 66\%

57. How many upper digestive endoscopies are performed in your unit with an anesthetist present? None; $<33 \%$; 33-66\%; > 66\% 


\section{Table I (Cont.)}

58. How many upper digestive endoscopies should be ideally performed in your unit with an anesthetist present? None: < 33\%; 33-66\%; > 66\%

59. How many colonoscopies are performed in your unit with an anesthetist present?

None; $<33 \%$; 33-66\%; > 66\%

60. How many colonoscopies should be ideally performed in your unit with an anesthetist present?

None; <33\%; 33-66\%; > 66\%

61. What kind of drug is normally used to sedate patients without an anesthetist present in your unit? Choose one or more: Midazolam; Dolantine; Propofol; Others; Local spray

62. What kind of drug do you consider ideal, without an anesthetist present, for the sedation of patients treated in your unit? Choose one or more: Midalozam; Dolantine; Propofol; Others; Local spray

63. What kind of drug is normally used, with an anesthetist present, to sedate patients in your unit? Choose one or more: Midazolam; Dolantine; Propofol; Others; Local spray

64. What kind of drug do you consider ideal, with an anesthetist present, for the sedation of patients treated in your unit? Choose one or more: Midazolam; Dolantine; Propofol; Others; Local spray

65. Is the administration of propofol monitored by an anesthetist? No; Sometimes; Yes

66. Do you think propofol should be administered by an anesthetist, or is it unnecessary?

No; Sometimes; Yes

67. How many days is there an anesthetist available in your unit per week? None; One; Two; Three or more

68. How many days there should ideally be an anesthetist available in your unit per week? None; One; Two; Three or more

69. Is the necessary material for patient sedation and recuperation (oxygen, pulse oximeter, vasoactive drugs, Ambu, laryngoscope) available in your unit? No; Yes

70. Do you think it necessary for your unit to have all the necessary material for sedation and recuperation (oxygen, pulse oximeter, vasoactive drugs, Ambu, laryngoscope) available? No; Yes

71. Is there a defibrillator in your unit? No; Yes

72. Do you think a defibrillator is necessary? No; Yes

Section 5: accessory material in the unit

73. Do you have an operating room with the conditions required for special examinations? No: Yes

74. Do you think that your unit should have a room with the conditions required for special examinations? No; Yes

75. Does your unit have an adequately equipped room for patient recovery from or emergencies during anesthesia? No; Yes

76. Do you think your unit should have an adequately equipped room for patient recovery from or emergencies during anesthesia? No; Yes

77. Does your unit have a proper waiting room for patient relatives? No; Yes

78. Do you think your unit should have a proper waiting room for patient relatives?

No; Yes

79. Does your unit have a proper office? No; Yes
80. Do you think your unit should have a proper office?

No; Yes

81. Is there an appropriate archive for documents and endoscopic images? No; Yes

82. Do you think your unit should have an appropriate archive for documents and endoscopic images?

No; Yes

Section 6: other

83. What are typically the shifts for endoscopists in your unit? Morning; Afternoon; Morning and afternoon

84. What is, in your opinion, the most suitable shift for the efficiency of endoscopists in your unit?

Morning; Afternoon; Morning and afternoon

85. How many endoscopic emergencies are treated every day in your endoscopy unit?

One; Two; Three; Four or more

86. What would be the ideal number of daily endoscopic emergencies for your unit?

One; Two; Three; Four or more

87. Are gastroenterologists to treat emergencies present or conveniently localized?

Present; Conveniently localized

88. How do you think gastroenterologists should proceed when on duty? Being present; Being conveniently localized

89. Is there any personnel (nurses and healthcare assistants) specialized in endoscopic emergencies?

No; Sometimes; Yes

90. Do you think it necessary to have personnel (nurses and healthcare assistants) specialized in endoscopic emergencies?

No; Sometimes; Yes

Open question number 91: Which aspects of endoscopy units should be improved for an optimum practice?

\section{RESULTS}

\section{Section 1: general questions}

Question no. 1. Answer: 4 hospitals cover a population with more than 400,000 people; 9 cover between 250,000 and 400,000; and 1 between 100,000 and 250,000.

Question no. 2. Answer: 3 hospitals think more than 400,000 is an ideal number of people to cover; 5 hospitals think between 250,000 and 400,000; and 6 think between 100,000 and 250,000 .

Question no. 3. Answer: in all hospitals endoscopy units are a part of gastroenterology departments.

Question no. 4. Answer: $84.6 \%$ would continue the same way, and only $16.4 \%$ would set up independent endoscopy units.

\section{Section 2: unit staff}

Question no. 5. Answer: 4 hospitals have between 5 and 10 doctors; 6 , between 10 and 15; and 4 have more than 15 doctors.

Question no. 6: Answer: 3 hospitals answered 5-10 doctors; 5, between 10 and 15; 6, more than 15 doctors.

Question no. 7: Answer: 10 hospitals have more than $66 \%$ of doctors working full-time; 2, between 33 and $66 \%$; and 1 fewer than $33 \%$; in one hospital no doctor works full-time in the unit. 


\section{Section 1: general questions}

Questions 1-2
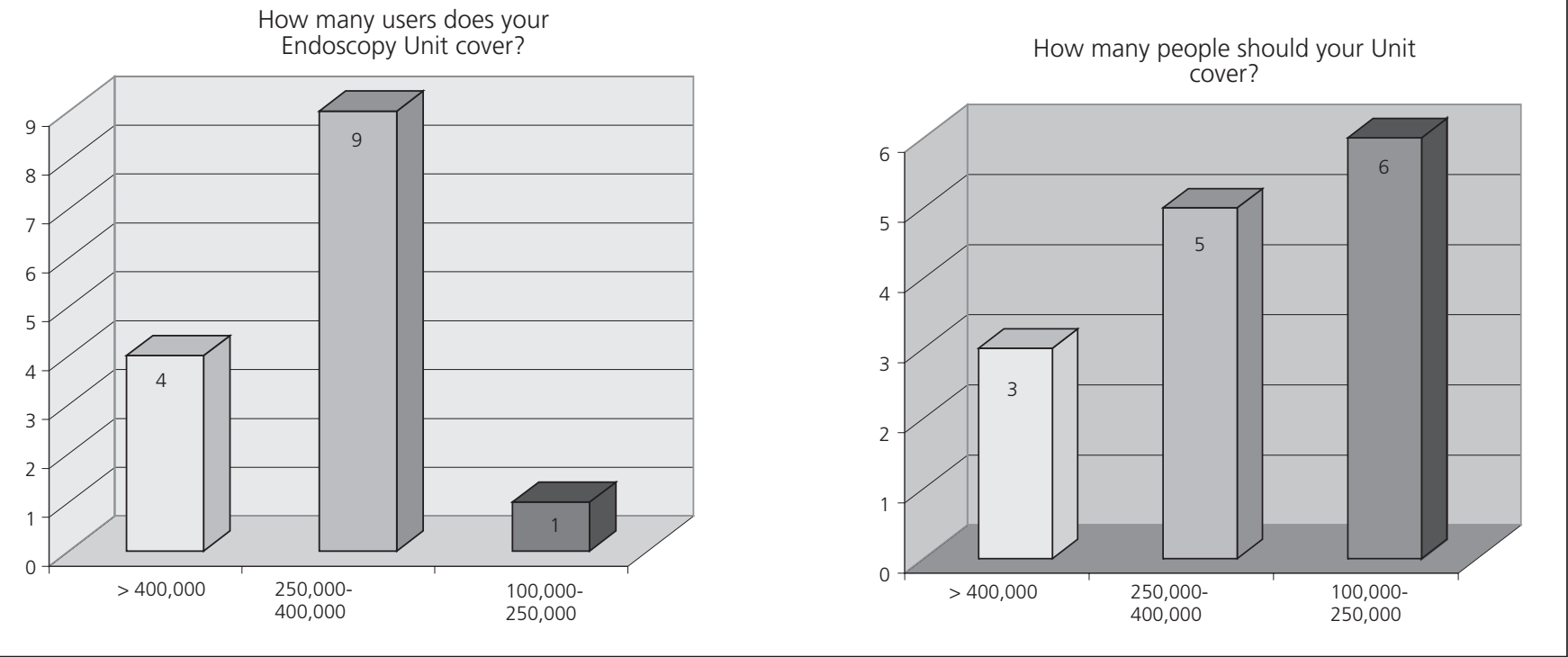

\section{Section 2: unit staff}

Questions 5-6
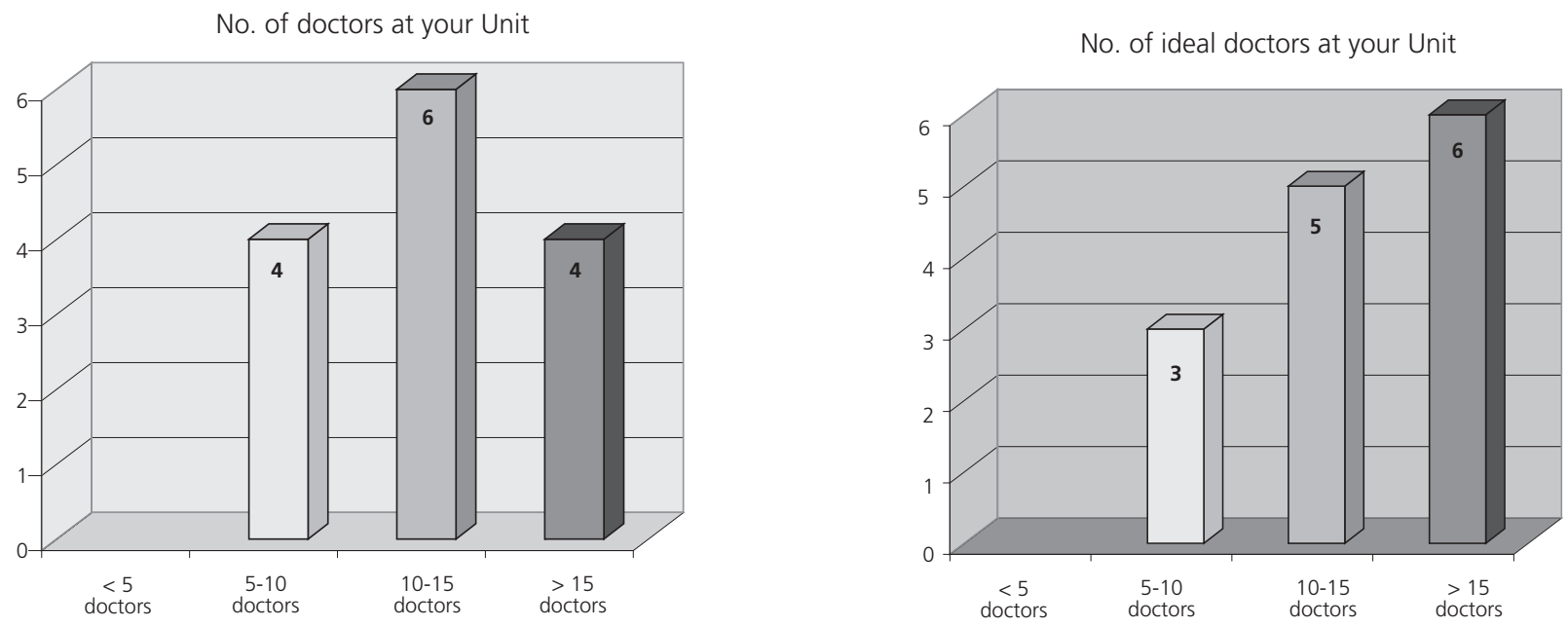

Question no. 8. Answer: 13 hospitals think that more than $66 \%$ of doctors should be working fulltime, while one hospital answered that $33 \%$ would suffice.

Question no. 9. Answer: 2 hospitals have fewer than 2 nurses; 8 , between 2 and 4; 3, between 4 and 6 ; 1 hospital has more than 6 nurses.

Question no. 10. Answer: 2 hospitals think it ideal to have fewer than 2 nurses; 6 , between 2 and 4 nurses; 5 hospitals, between 4 and 6 ; and 1 hospital, more than 6 nurses.
Question no. 11. Answer: 2 hospitals have fewer than 2 healthcare assistants; 7 have between 2 and 4; 4 hospitals have 4-6; and 1 hospital has more than 6 healthcare assistants.

Question no. 12. Answer: no hospital answered that less than 2 auxiliary nurses would be ideal; 8 hospitals answered 2-4 would be ideal; 5 hospitals think 4-6 healthcare assistants would be enough, and 1 hospital answered that the ideal number would be more than 6 . 


\section{Section 3: unit material}

Questions 15-16
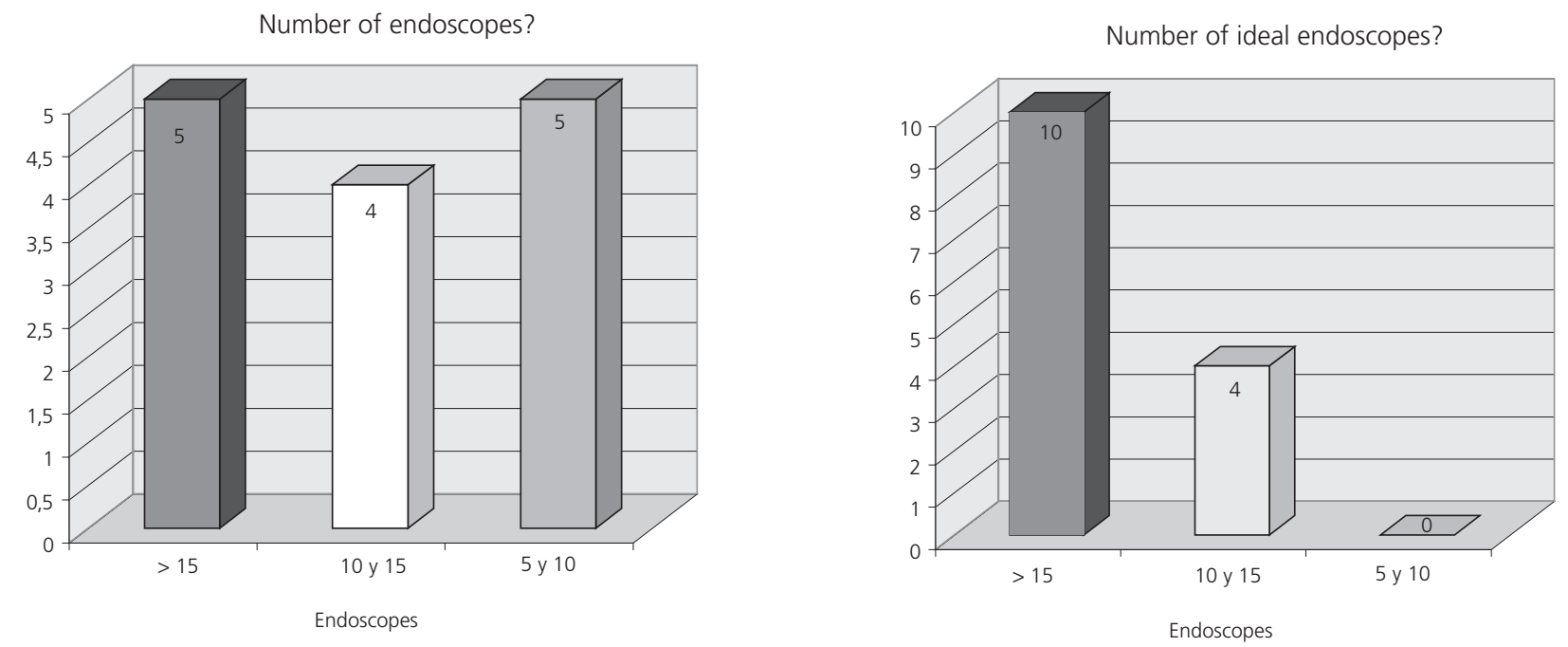

\section{Section 3: unit material}

Questions 27-28
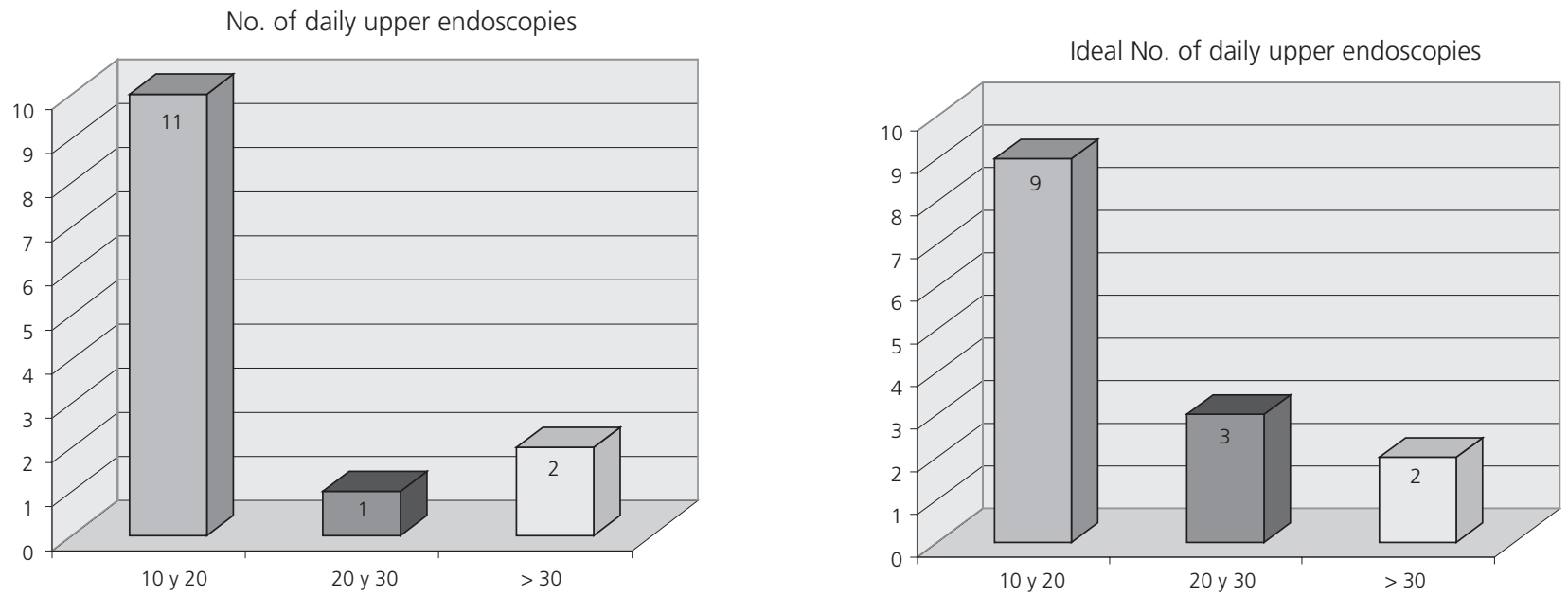

\section{Section 3: unit material}

Question no. 13. Answer: in two hospitals the number of rooms is 2 ; in seven hospitals there are 3 rooms, and in 5 hospitals there are four or more rooms.

Question no. 14. Answer: four hospitals answered that the ideal number of rooms is $3 ; 10$ of them think that four or more would be ideal.

Question no. 15. Answer: in 5 hospitals the number of endoscopes is higher than 15; in four of them there are between 10 and 15; in 5, there are 5-10 endoscopes.

Question no. 16. Answer: 10 hospitals answered that the ideal number of endoscopes would be more than 15; 4 of them consider that 10-15 would be enough.

Question no. 17. Answer: 4 hospitals have between 2 and $4 ; 4$ of them have 4-6; and 6 hospitals have more than 6 gastroscopes.

Question no. 18. Answers: 5 hospitals answered that the ideal number of gastroscopes would be 4-6; 9 of 


\section{Section 4: sedation}

Questions 53-54
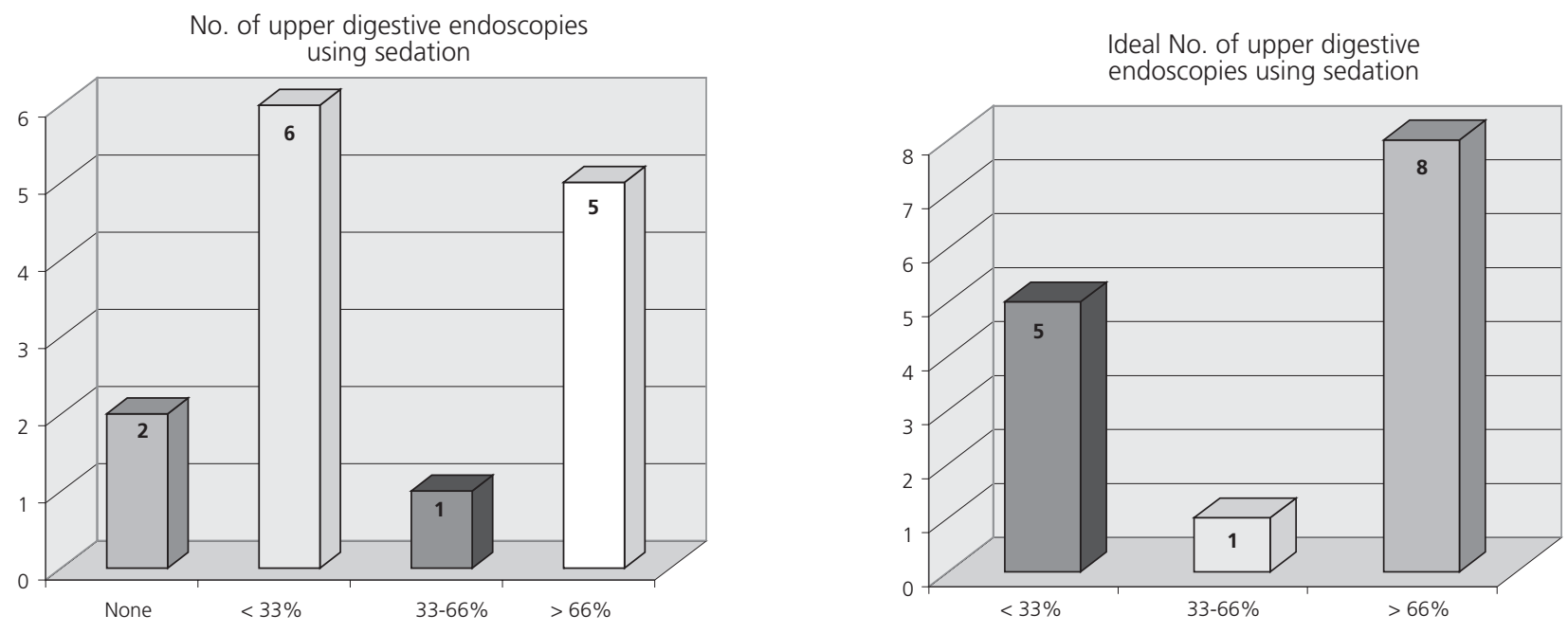

\section{Section 4: sedation}

Questions 67-68
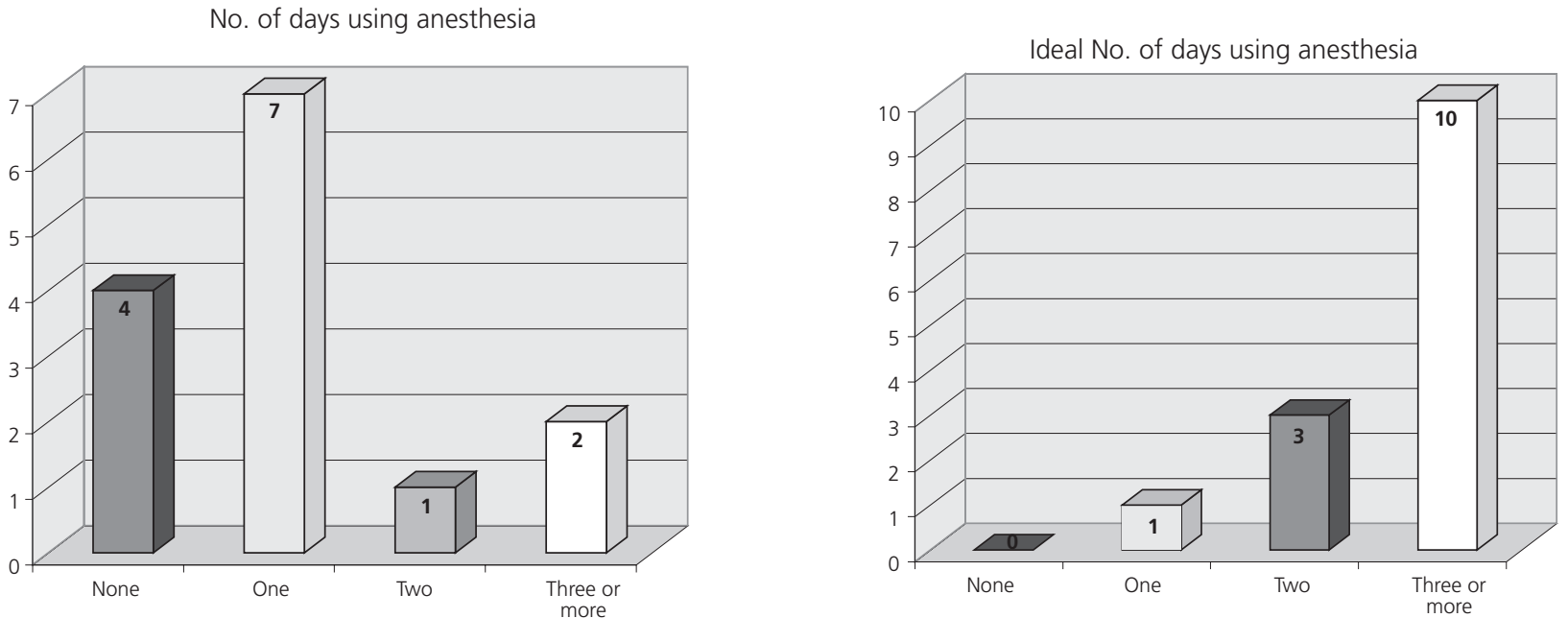

them think that more than 6 would be the right amount.

Question no. 19. Answers: 3 hospitals have 2-4 colonoscopes; 4 , between 4 and 6 ; and 6 hospitals have more than 6 colonoscopes.

Question no. 20. Answer: 6 hospitals think the ideal number of colonoscopes is 4-6; 8 hospitals answered the ideal number is more than 6.

Question no. 21. Answer: 3 hospitals have 1 duodenoscope; 7 of them have 2; 3 of them have 3 and 1 hospital has 4 or more.
Question no. 22. Answer: 5 hospitals think the ideal number of duodenoscopes is 2; 3 hospitals answered that 3 would be enough; and 6 think 4 or more.

Question no. 23. Answer: 9 hospitals have no enteroscopes, and 5 hospitals have 1.

Question no. 24. Answer: 10 hospitals consider it ideal to have 1 enteroscope, and 4 think 2 is the ideal number.

Question no. 25. Answer: 6 hospitals have no echoendoscopes; 6 hospitals have 1; and 2 hospitals have 2 .

Question no. 26. Answer: 4 hospitals answered that 
they should have 1; 7 think they should have 2; and 3 hospitals think they should have three or more.

Question no. 27. Answer: 11 hospitals do 10-20 gastroscopies every day: 1 hospital, 20-30; and 2, more than 30.

Question no. 28. Answer: 9 hospitals answered that they should do 10-20 every day; 3, 20-30; and 2, more than 30 .

Question no. 29. Answer: 2 hospitals do fewer than 10 colonoscopies every day; 8 , between 10 and 20; 3 hospitals do 20-30; and 1 hospital does more than 30 every day.

Question no. 30. Answer: 2 hospitals answered that they should do fewer than 10 every day; 4, between 10 and 20; 5, between 20 and 30; and 3 hospitals answered that they should do more than 30 every day.

Question no. 31. Answer: 9 hospitals do not do any enteroscopies; 3 of them do 1 per week; 1 of the hospitals does 2; and only one of the hospitals does three or more.

Question no. 32. Answer: 2 hospitals answered that they should not do any enteroscopies; 6 think they should do 1; 3 of them, 2 enteroscopies; and 3 hospitals think they should do three or more.

Question no. 33. Answer: three hospitals do 2 per week; the other 11 do three or more.

Question no. 34. Answer: 1 hospital answered that the ideal number depends on individual needs; the other 13 think that they should be doing three or more.

Question no. 35. Answer: 6 hospitals do not do any echoendoscopies; three hospitals do 2; and 5 hospitals do three or more.

Question no. 36. Answer: two hospitals answered that they should be doing 1 echoendoscopy; three of them, 2; and 9 , three or more.

Question no. 37. Answer: in one hospital they do not give appointments for polypectomies specifically, but polypectomies are done when necessary. The other 13 hospitals do three or more.

Question no. 38. Answer: All hospitals answered that 3 or more should be done.

Question no. 39. Answer: 4 hospitals do one treatment; 3 of them do 2; and 7 do three or more.

Question no. 40. Answer: 2 hospitals answered that no varicose vein treatment should be done; 3 of them answered that only one per week; 5 hospitals said two; and 4 , three or more.

Question no. 41. Answer: no hemorrhoidal treatment is carried out in ten hospitals; in one of them, one treatment is done per week; one hospital does two; and two of them do three or more.

Question no. 42. Answer: 7 hospitals think that no hemorrhoidal treatments should be done; one hospital answered that 1 per week; 2 hospitals, 2 per week; and 4 hospitals, three or more.

Question no. 43. Answer: 1 hospital does no treatments with argon beam; two hospitals do 1 treatment per week; 2 of them do two; 9 hospitals do three or more.
Question no. 44. Answer: two hospitals answered that no argon beam treatment should be done; two answered that one per week; 1, two per week; 9 , three or more.

Question no. 45. Answer: one of the hospitals does not do this kind of treatment; 4 of them do 1 per week; 1 does two; 8 hospitals do three or more.

Question no. 46. Answer: three hospitals answered that they should do one treatment per week; 1 hospital answered that 1 treatment; 9 hospitals answered that three or more; 1 hospital thinks that no treatments should be done.

Question no. 47. Answer: 5 hospitals take less than 15 minutes; 6 of them, 15-25 minutes; 3 of them, 25-35 minutes.

Question no. 48. Answer: 3 hospitals think that the ideal time is less than 15 minutes; 8 of them think that 15-25 minutes; 3 hospitals, between 25 and 35 minutes.

Question no. 49. Answer: the estimated time in one of the hospitals is shorter than 25 minutes; in 9 hospitals it is 25-40 minutes; in 3, 40-55 minutes; in one of them, more than 55 minutes.

Question no. 50. Answer: 1 hospital answered that the estimated time should be shorter than 25 minutes; 8 , between 25 and 40 minutes; 5 of them, $40-55$ minutes.

Question no. 51. Answer: 2 hospitals set the time below 40 minutes; 8 of them, $40-70$ minutes; 3 of them, 70100 minutes; 1 hospital, more than 100 minutes.

Question no. 52. Answer: two hospitals answered that less than 40 minutes would be enough; 8 of them, 40-70 minutes; and 4 hospitals, 70-100 minutes.

\section{Section 4: sedation and anesthesia}

Question no. 53. Answer: in two hospitals they do no gastroscopies with sedation; in 6 of them, less than $33 \%$; in one of them, 33-66\%; in 5 hospitals, more than $66 \%$.

Question no. 54. Answer: 5 hospitals answered that sedation should not be used; one of the hospitals answered that $33-66 \%$; 8 of them, more than $66 \%$.

Question no. 55. Answer: in one hospital none is done with sedation; in 4 of them, less than 33\%; in two hospitals, between 33 and $66 \%$; in 7 of them, more than $66 \%$.

Question no. 56. Answer: three hospitals answered that $33-66 \%$; 11 of them answered that more than $66 \%$.

Question no. 57. Answer: none is done using anesthesia in 5 hospitals; in 9 hospitals, less than $33 \%$.

Question no. 58. Answer: 2 hospitals answered that no gastroscopy should be done using anesthesia; 6 answered less than $33 \%$; 4 of them answered 33-66\%; one hospital thinks more than $66 \%$.

Question no. 59. Answer: in three hospitals colonoscopies are done without anesthesia; in 10 hospitals, less than $33 \%$; in 1 of them, $33-66 \%$.

Question no. 60. Answer: 1 hospital answered that they should not do any; 3 of them, less than 33\%; 3 of the hospitals, between 33 and $66 \%$; and 7, more than $66 \%$.

Question no. 61. Answer: 7 hospitals use midazolam, Dolantine and local anesthesia; 5 use midazolam and Dolantine; 1 uses midazolam plus local anesthesia; and one uses only local anesthesia. 
Question no. 62. Answer: 7 hospitals answered that midazolam and Dolantine are the ideal drugs; 4 hospitals add a local anesthetic to these two; 2 chose midazolam and Dolantine; 1 hospital answered that the chosen drug should be propofol.

Question no. 63. Answer: in 9 hospitals the most frequently used drug is propofol; in two of them, propofol together with fentanyl; in other two, propofol together with other drugs; in one, propofol together with Dolantine.

Question no. 64. Answer: 10 hospitals answered that the ideal drug is propofol; one of the hospitals, propofol in combination with fentanyl; other hospital, in combination with Dolantine; 2 hospitals preferred a combination of propofol and drugs other than these.

Question no. 65. Answer: in 100\% of cases anesthetists monitor the process.

Question no. 66. Answer: $93 \%$ think it necessary; only $7 \%$ think it unnecessary.

Question no. 67. Answer: 4 hospitals have no anesthetist available; 7 hospitals have an anesthetist one day per week; one hospital, two days; 2 hospitals, three or more.

Question no. 68. Answer: 1 hospital answered that it is enough with an anesthetist one day a week; 3, two days; the other ten hospitals, three or more days.

Question no. 69. Answer: $14 \%$ of hospitals do not have such resources; $86 \%$ do.

Question no. 70. Answer: $100 \%$ of hospitals think these resources are necessary.

Question no. 71. Answer: $29 \%$ of hospitals have a defibrillator; $14 \%$ of hospitals surveyed did not answer of did not know the right answer; $57 \%$ do not have this material.

Question no. 72. Answer: $71 \%$ think it necessary; $29 \%$ do not.

\section{Section 5: accessory material in the unit}

Question no. 73. Answer: 50\% of hospitals do not have this kind of room; $50 \%$ do.

Question no. 74. Answer: $100 \%$ of hospitals think these rooms are necessary.

Question no. 75. Answer: $36 \%$ of hospitals have this kind of room; $64 \%$ do not.

Question no. 76. Answer: $100 \%$ of them think it necessary.

Question no. 77. Answer: $57 \%$ do not have a waiting room; 43 do have it.

Question no. 78. Answer: $100 \%$ of hospitals think they should have one.

Question no. 79. Answer: half of hospitals contacted do not have an office; the other $50 \%$ have one.

Question no. 80. Answer: $100 \%$ of hospitals think that one is necessary.

Question no. 81. Answer: $71 \%$ of units do not have an archive; $29 \%$ of them have one.

Question no. 82. Answer: $93 \%$ of them answered that they should have one; only $7 \%$ said no.

\section{Section 6: other matters}

Question no. 83. Answer: $43 \%$ of units operate in the morning and afternoon; $57 \%$ of them have a morning shift.

Question no. 84. Answer: half of units answered that a morning shift is ideal; the other half answered that it is appropriate to work in the morning and the afternoon.

Question no. 85. Answer: 2 hospitals usually do 2 emergencies every day; 8 hospitals do $3 ; 4$ of them do four or more.

Question no. 86. Answer: two hospitals answered that 2 emergencies would be ideal; 8 answered 3; 4 hospitals, four or more.

Question no. 87. Answer: $38 \%$ of gastroenterologists are localized when on duty; $57 \%$ are present. In one hospital there are not any gastroenterologists on duty.

Question no. 88. Answer: $43 \%$ think that doctors on duty should be conveniently located; $57 \%$ think that they should be present.

Question no. 89. Answer: 58\% sometimes have specialized personnel; $21 \%$ do not have any; $21 \%$ have it.

Question no. 90. Answer: $93 \%$ of units think it necessary to have specialized personnel.

Question no. 91. This is an open question and consequently each unit or department provided their view on how they could improve. However, a few aspects have been mentioned by most hospitals questioned: first, the need to have anesthetists more frequently; then, a higher number of endoscopes and other material, as well a having an RX column and staff specialized in several fields.

\section{OTHER FEATURES ASSESSED}

The objective was to find out the variety of techniques used, and the number of endoscopes per 100,000 people. The number of people cared for by these units has been rounded off. The number of people assisted by units covering 400,000 people has been rounded up to 500,000; numbers between 250,000 and 400,000 have been rounded off to 300,000 ; and figures between 100,000 and 250,000 , to 200,000. Multiplied by the number of units, we obtain $4,900,000$. A similar procedure has been carried out with the number of both techniques and endoscopes.

The average number of endoscopes per 100,000 people in units as a whole is 2.46. The number of gastroscopes is 1.63 per 100,000 inhabitants. There are 1.57 colonoscopes, 0.63 duodenoscopes, 0.1 enteroscopes, and 0.20 echoendoscopes per 100,000 population.

The average number of gastroscopies performed every day is 5.30 per 100,000 people. The number of colonoscopies is $9.79 ; 0.22$ enteroscopies are performed every week, 1.46 weekly ERCPs, 0.75 echoendoscopies, 1.6 polypectomies, 1.06 endoscopic treatments of varicose veins, 0.22 hemorrhoidal treatments, 0.91 argon beam treatments, and 0.75 palliative treatments are performed every week per 100,000 population. 


\section{DISCUSSION}

The present survey intends to cover all significant aspects in an endoscopic unit. Questions relate to materials, human resources, time devoted to each test, how tests are done, and other unit resources. The aim was to assess the current situation in them, and to find out what the ideal condition would be. However, it is difficult to compare these results since there is no other survey and there is no model to know how a unit should be organized or what materials should have.

Answers to the first two questions indicate a higher demand than desirable; 5 hospitals answered that they should care for fewer patients than they actually do. This means that some units cover a population larger than they should, considering the resources they have. May be this is one of the main problems to solve.

All endoscopy units in Andalusia are part of a gastroenterology department, and $84 \%$ of them think that this is the way it should be. The relationship between an endoscopy unit and the rest of a gastroenterology department is essential for their work to be efficient. We should bear in mind that most endoscopy patients come from outpatient clinics and the gastroenterology ward.

As to the staff working in most hospitals, answers show that a more numerous personnel are needed, including doctors, nurses and healthcare assistants. There are also questions in the survey about a unit's staff. Due to the increasing complexity of tests and treatments, the auxiliary staff should be especially trained. The efficiency of a unit is connected with the professional standards of its staff. With regard to doctors, 13 hospitals answered that they should have more doctors working full-time in the unit. The idea of specialization in the field of endoscopy is again stressed.

None of the hospitals answered that it was enough for a unit having less than 2 rooms; ten hospitals think that 4 or more rooms would be ideal. However, there are 2 hospitals included in the survey with only 2 rooms, and only 5 hospitals have 4 or more. The place where endoscopic techniques are carried out is also very important for unit efficiency. Fifteen years ago endoscopy units were just improvised rooms connected with emergency rooms. Today, improvising the setup of a unit would be unconceivable. There should be rooms for diagnostic endoscopies, and at least one for more complex, time-consuming techniques. Computers allow for a reduction of costs and greater efficiency.

Hospitals ask for more endoscopes. They consider 15 to be the minimum number required. It is remarkable that 9 hospitals have no enteroscopes, and 6 of these have no echoendoscopes. Obviously, the essential parts for these units are endoscopes. Material care and maintenance is basic if instruments are to last long. Again the idea of an expert staff for machine operation is posed. The amount of material should be proportional to the number of people covered by each unit. Thus, the results are as follows: the number of endoscopes per 100,000 people as covered by all units is 2.46 . Figures concerning gastroscopes are 1.63 per 100,000 people. There are 1.57 colonoscopes, 0.63 duodenoscopes, 0.1 enteroscopes, and 0.20 echoendoscopes per 100,000 people. The number of duodenoscopes, enteroscopes and echoendoscopes seems insufficient.

In general, the number of daily gastroscopies and colonoscopies seems adequate, though hospitals think that the number of weekly ERCPs and echoendoscopies should be raised. The number of gastroscopies done every day per 100,000 is 5.30 . The number of colonoscopies is 9.79; the number of enteroscopies is 0.24 every week. The number of ERCPs is 1.46 every week. The figure for echoendoscopies is 0.75 .

There is some agreement in the assessment of the time that each examination should take, so that the time actually devoted to these tests in each unit is considered to be correct. The ideal time for a gastroscopy is $15-25 \mathrm{~min}-$ utes, 25-40 minutes for a colonoscopy; for a non-basic examination, it is $40-70$ minutes. The time used for every examination is also questioned. In endoscopies efficiency can be measured in the number of endoscopies that one endoscopist can do over a certain period of time. However, the skill of the endoscopist in doing his or her job in a short time should not be the most important factor. The article by Zamir et al. shows the results of questions posed to doctors and chief nurses about the most important factors in an efficient endoscopy unit. Their answer referred to the number of rooms for endoscopies, number of nurses, and a room for the patient recovery. The speediness of the doctor doing the technique was fifth.

The group of questions about sedation deals with aspects that are very important today, as it is very topical. The number of techniques and examinations requiring anesthesia, and an anesthetist, is growing. However, a rise in the number of specialists has not been promoted. Endoscopies, even therapeutic endoscopies, are classified as low-risk, but quality and most of all safety must be always the rule. Endoscopy is regarded as an invasive but low-risk technique by most endoscopy units, and as a consequence most endoscopists perform sedation for their patients.

Some medical and legal aspects are noted in this: because of the invasive character of the technique, the patient must always sign an informed consent.

The question of who should sedate patients has been dealt with in articles and protocols by many scientific societies. The protocols of the American Society of Anesthesiologists (ASA) recommend that a specialist different from the one who is going to perform the endoscopy should monitor and watch the patient. Every time propofol is used there should be a specialist present to recover the patient from general anesthesia.

A prospective, descriptive study has been recently published based on the making, distribution, and analysis of a survey about clinical practice in Galician hospitals. 
The information asked concerned examinations, resources available for sedation, means to control sedation, and level of sedation used and desirable for each examination. Twenty endoscopy units provided their answers -thirteen were in public hospitals and eleven used to perform highly complex examinations.

The results about resuscitation materials and special rooms for anesthesia recovery are quite similar to those in the present survey: $80 \%$ of units had pulse oximetry; $86 \%$ of hospitals present in this survey had resuscitation means. However, there is a shortage of sedation material in two of the hospitals asked, of rooms for special examinations in half of them, and for anesthesia recovery in $64 \%$ of hospitals. All hospitals answered that an office and a waiting room are necessary, and 13 claimed that an archive is important.

Forty-two percent of Galician units have electrocardiographic monitoring. Our survey does not include specifically this matter, but the material mentioned in question 69 is thought to include such monitoring. Only $29 \%$ of hospitals have a defibrillator. In Galicia, $40 \%$ of hospitals have a defibrillator, and $45 \%$ have a recovery room. In Andalusia, 36\% of hospitals have such a room.

The survey in Andalusia does not show much coincidence as to the need for anesthesia in colonoscopies and gastroscopies. The most frequently used drug is propofol, which must be monitored by an anesthetist. It is important to mention that 11 hospitals do not have an anesthetist available, or only have one 1 day per week. Thirteen hospitals think that anesthetists should be on duty two, three or more days a week. The survey from Galicia shows that an anesthetist was available for certain examinations in $15 \%$ of cases, and exceptionally in $65 \%$ of units. Thirty-five percent of Galician units think it is desirable to have an anesthetist full-time; $25 \%$, only for certain examinations, and $35 \%$, only exceptionally. In Catalonia, $7 \%$ of gastroscopies, $25 \%$ of colonoscopies, and $38 \%$ of ERCPs are always done with an anesthetist present. Anesthetists are being increasingly demanded by all units due to technique complexity, which requires a higher level of safety during examinations. Also because of such complexity, a doctor performing an endoscopy cannot monitor the patient concurrently.

In Andalusia, 8 of the surveyed hospitals either do not use sedation in gastroscopies or use it in less than $33 \%$ of cases. Nine of them think that more than $33 \%$ of gastroscopies should be done with sedation. There is not such a big difference regarding colonoscopies, as in most hospitals more than $33 \%$ are done with sedation. Midazolam and Dolantine are the most frequently used drugs.

In Galicia units answered that sedation is necessary in $83 \%$ of therapeutic gastroscopies, in $87 \%$ of therapeutic colonoscopies, in $98 \%$ of ERCPs, in $95 \%$ of enteroscopies, and in $98 \%$ of echoendoscopies. The most frequent drug used in gastroscopies was midazolam (76\%), and the combination midazolam/meperidine was the most frequently used sedation during colonoscopies (72\%) and ERCPs (60). In Catalonia, 17\% of gastroscopies, $61 \%$ of colonoscopies, and $100 \%$ of ERCPs are performed using sedation. The Galician survey shows that while endoscopists think that most endoscopic techniques must be done using sedation, some units do not have the necessary means to safely monitor their patients. These results are not very different from those of our survey. Sedating drugs are the same as those used in our units. Galician endoscopists also coincide with us in that invasive techniques should be done using a higher degree of sedation or anesthesia, monitored by an anesthetist.

Our survey also deals with working shifts. There are not many differences as to shifts, number of emergencies, or how duties are done. It is remarkable, though, that $93 \%$ of hospitals think it necessary to have specialized personnel in the casualties department.

\section{CONCLUSIONS}

This survey shows that, while some things need to be improved, a high percentage of hospitals have acceptable basic means. However, due to the fast rate of advance in the field of endoscopy, improvement is needed especially regarding anesthesia and modern material.

\section{REFERENCES}

1. Faigel DO, Baron TH, Goldstein JL, Hirota WK, Jacobson BC, Johanson JF, et al, Standards Practice Committe, American Society for Gastrointestinal Endoscopy. Guidelines for the use of deep sedation and anesthesia for GI endoscopy. Gastrointest Endosc 2002; 56 (5): 613-7.

2. Karan SB, Bailey PL. Update and review of moderate and deep sedation. Gastrointest Endosc Clin N Am 2004; 14 (2): 289-312.

3. Lazzaroni M, Bianchi Porro G. Preparation, premedication, and surveillance. Endoscopy 2005; 37(2): 101-9.

4. Cubiella Fernández J, Lancho Seco A, Echarri Piudo, et al. Sedation at Endoscopic Units in Galicia: Results of the "Sociedad Gallega de Patología Digestiva" inquiry. Rev Esp Enferm Dig 2005; 97: 24-37.

5. Campo R, Brullet E, Junquera F, Puig-Divi V, Vergara M, Montserrat A, et al. Sedación y anestesia en endoscopia digestiva. Resultados de una encuesta hospitalaria en Cataluña. Gastroenterol Hepatol 2004; 27 (3): 205.

6. Ginsberg GG. Intraprocedural steps to enhance efficiency. Gastrointest Endoscopy Clin N Am 2004; 14: 635-45.

7. Kowalski T, Edmundowicz, S, Vacante N. Endoscopy unit form and function. Gastrointest Endoscopy Clin N Am 2004; 14: 657-66.

8. Elta G H. Efficiency in endoscopy with improvements in instrumentation. Gastrointest Endoscopy Clin N Am 2004; 14: 673-8.

9. Dunkin BJ. Lessons learned from the operating room about procedure efficiency. Gastrointest Endoscopy Clin N Am 2004; 14: 667-72.

10. Zamir S, Rex DK. An initial investigation of the efficiency in endoscopy delivery. Am J Gastroenterol 2002; 97: 1968-72.

11. López Timoneda F, Ramírez Armengol. Who should be responsible for sedation techniques in digestive endoscopy? Rev Esp Enferm Dig 2005; 97: 1-6. 